Geometry $8 \mathcal{F}$ Topology

Volume 4 (2000) 219-242

Published: 12 September 2000

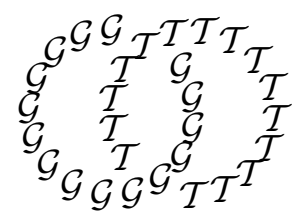

\title{
Tight contact structures and taut foliations
}

\author{
KO HONDA \\ WILLIAM H KAZEZ \\ Gordana Matić \\ Mathematics Department, University of Georgia \\ Athens, GA 30602, USA \\ Email: honda@math.uga.edu, will@math.uga.edu, gordana@math.uga.edu

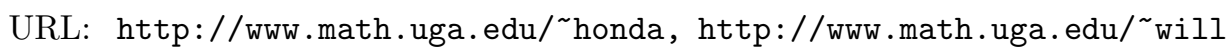

\begin{abstract}
We show the equivalence of several notions in the theory of taut foliations and the theory of tight contact structures. We prove equivalence, in certain cases, of existence of tight contact structures and taut foliations.
\end{abstract}

AMS Classification numbers Primary: 57M50

Secondary: $53 \mathrm{C} 15$

Keywords: Tight, contact structure, taut foliation

Proposed: Yasha Eliashberg

Received: 21 April 2000

Seconded: Robion Kirby, David Gabai

Revised: 20 July 2000

Copyright Geometry and Topology 


\section{Introduction}

The goal of this paper is to relate aspects of the theory of taut foliations and the theory of tight contact structures. Codimension- 1 foliations of 3 -manifolds have a rich and beautiful history. Highlights include the first examples on $S^{3}$, due to Reeb, Haefliger's proof of the non-existence of analytic foliations on $S^{3}$, and Novikov's proof of the necessity of Reeb components in foliations of $S^{3}$. As a result of Gabai's work, the class of foliations that have played the most important role in 3-dimensional topology, and especially in knot theory, are the taut foliations. The theory of tight contact structures, on the other hand, has not yet reached a phase where it can be applied effectively to the study of the topology of 3-manifolds. It is still concerned with basic questions about the structures themselves, such as existence and classification on even some of the simplest manifolds, such as handlebodies. The classification on $S^{3}$ and $B^{3}$ is due to Eliashberg in 1991, and the classification on $T^{3}$ has been known only since $1995[21,13]$. Only recently has the classification been completed for lens spaces $L(p, q)[14,17]$ and has the first example of a manifold with no tight contact structure been produced [9] (the Poincaré homology sphere $\Sigma(2,3,5)$ with one of its orientations).

Any relationship between these structures is not only interesting in its own right, but also provides hope and an indication that contact structures will become a valuable tool for studying 3-dimensional topology. Eliashberg and Thurston [10] bridged the gap between foliation theory and contact topology. Their seminal work opened the door and enabled an exchange of ideas between two neighboring fields. They proved that if a 3-manifold carries a taut foliation, then it also supports a tight contact structure (in fact, one for each orientation of the ambient manfold $M$ ). Although their method of perturbing a foliation into a contact structure is 3 -dimensional, their method of proving tightness is not 3-dimensional, and instead uses the results from 4-dimensional symplectic topology on symplectic fillings. In [19] we reprove, and partially extend, their theorem using purely 3 -dimensional techniques. The purpose of this paper is to prove a converse, in the case of a 3-manifold with boundary, namely that if it supports a tight contact structure, it supports a taut foliation. Note that we cannot hope to prove the converse in the case of a general closed manifold, since there are simple examples, like $S^{3}$, which support tight contact structures but carry no taut foliations.

The techniques we use are based on a Haken decomposition theory, where the cutting manifolds are convex surfaces. In Section 2 of this paper, we briefly explain the notion of a convex surface in a contact manifold as introduced by 
Giroux [12]. These appear to us to be the best kind of cutting surface for a decomposition of a manifold with a contact structure. In Section 3, we explain how to perturb a convex surface and a (not necessarily Legendrian) curve $\gamma$ on it, so that $\gamma$ becomes Legendrian. In Section 4 we will explain how to cut along convex surfaces with Legendrian boundary to perform a convex splitting on $M$. These will be used to cut the manifold eventually down to a union of balls. Each ball supports a unique tight contact structure up to isotopy rel boundary, by a fundamental theorem of Eliashberg [7]. The contact structure on $M$ is therefore encoded in the splitting surfaces $S$ together with characteristic foliation on $S$. Moreover, the characteristic foliation on a convex surface $S$ is better encoded by a collection of curves called the dividing set $\Gamma_{S}$. Abstracting the idea of a 3-manifold $M$ with 'curved' boundary $(\partial M, \Gamma)$ ( $\Gamma$ is a collection of curves), we define the notion of a convex structure. This notion closely resembles the notion of sutured manifolds introduced by Gabai [11] which we will recall in Section 5. Gabai used sutured manifold decompositions to construct taut foliations. We will show that a convex Haken decomposition is, in a sense, a generalization of a sutured manifold decomposition, and that the existence of a tight contact structure on a manifold with given convex structure on the boundary implies the existence of a taut foliation with the corresponding sutured manifold structure. Our main result, which incorporates important results of Gabai, Thurston and Eliashberg is:

Theorem 6.1 Let $(M, \gamma)$ be an irreducible sutured manifold with annular sutures, and let $(M, \Gamma)$ be the associated convex structure. The following are equivalent.

(1) $(M, \gamma)$ is taut.

(2) $(M, \gamma)$ carries a taut foliation.

(3) $(M, \Gamma)$ carries a universally tight contact structure.

(4) $(M, \Gamma)$ carries a tight contact structure.

\section{Convex surfaces and convex structures}

Let $M$ be an oriented, compact 3-manifold (possibly with boundary). A cooriented positive contact structure on $M$ is a nowhere integrable 2-plane field $\xi \subset T_{*} M$ such that there is a global 1 -form $\alpha$ for which $\alpha \wedge d \alpha=f \Omega$ with $f>0$ and $\Omega$ a volume form, and for which $\xi=\operatorname{ker} \alpha . \alpha$ determines the orientation of $\xi$. A curve that is everywhere tangent to the contact structure $\xi$ is 
called Legendrian. If $\Sigma$ is an embedded surface, $\xi$ induces on it the characteristic foliation $\left.\xi\right|_{\Sigma}$, which is defined to be the singular foliation consisting of the integral curves of $\xi \cap T_{*} \Sigma$ on $\Sigma$. Clearly these integral curves are Legendrian.

A contact structure $\xi$ is said to be overtwisted if there exists a disk $D$ which is everywhere tangent to $\xi$ along the boundary. Such a disk $D$ is called an overtwisted disk. A contact structure $\xi$ which is not overtwisted is said to be tight. Eliashberg [4] showed that, for closed 3-manifolds, the set of overtwisted contact 2-plane fields is weak homotopy equivalent to the set of contact 2-plane fields (without any integrability conditions). Hence, the study of overtwisted contact structures is largely homotopy-theoretic (of course there is the problem of determining whether a contact structure is tight or overtwisted). Tight contact structures are less ubiquitous, and tend to reflect the topology of the $3-$ manifold in ways which are not very well-understood.

We say a vector field $v$ on a contact manifold $(M, \xi)$ is a contact vector field if its flow preserves $\xi$. An oriented properly embedded surface $\Sigma$ in $(M, \xi)$ is called convex if there is a contact vector field $v$ transverse to $\Sigma$. The dividing set $\Gamma_{\Sigma}$ of a convex surface $\Sigma$ with respect to a transverse contact vector field $v$ is the set of points $x$ for which $v(x) \in \xi(x)$. The following is a fundamental theorem of Giroux [12].

Theorem 2.1 (Giroux [12]) The dividing set $\Gamma_{\Sigma}$ is a union of smooth curves which are transverse to the characteristic foliation $\left.\xi\right|_{\Sigma}$. Moreover, the isotopy type of $\Gamma_{\Sigma}$ is independent of the choice of $v$.

The isotopy class of $\Gamma_{\Sigma}$ is clearly preserved under an isotopy of $\Sigma$ through a family of convex surfaces. Conversely, if $\mathcal{F}$ is a singular foliation on $\Sigma$, then a disjoint union of properly embedded curves $\Gamma$ is said to divide $\mathcal{F}$ if there exists an $I$-invariant contact structure $\xi$ on $\Sigma \times I$ such that $\mathcal{F}=\left.\xi\right|_{\Sigma \times\{0\}}$ and $\Gamma$ is the dividing set for $\Sigma \times\{0\}$.

Denote the number of connected components of $\Gamma_{\Sigma}$ by $\# \Gamma_{\Sigma}$. The complement of the dividing set is the union of two subsets $\Sigma \backslash \Gamma_{\Sigma}=R_{+}-R_{-}$. Here $R_{+}$ is the subsurface where the orientations of $v$ and the normal orientation of $\xi$ coincide, and $R_{-}$is the subsurface where they are opposite. If $\Sigma$ is a surface with boundary, in this paper we also require that the boundary be a Legendrian curve for $\Sigma$ to be called convex.

Theorem 2.2 (Giroux's Flexibility Theorem [12]) Let $\Sigma$ be a convex surface in a contact 3 -manifold $(M, \xi)$ with characteristic foliation $\left.\xi\right|_{\Sigma}$, contact vector field $v$, and dividing set $\Gamma$. If $\mathcal{F}$ is another singular foliation on $\Sigma$ divided 
by $\Gamma$, then there is an isotopy $\phi_{t}: \Sigma \rightarrow M, t \in[0,1]$, such that $\phi_{0}(\Sigma)=\Sigma$, $\left.\xi\right|_{\phi_{1}(\Sigma)}=\mathcal{F}$, the isotopy is fixed on $\Gamma$, and $\phi_{t}(\Sigma)$ is transverse to $v$ for all $t$.

Such an isotopy is said to be an admissible isotopy of a convex surface $\Sigma$ with respect to a contact vector field $v \pitchfork \Sigma$. If the contact vector field $v$ is omitted, it is implied that the isotopy is admissible with respect to some $v$.

Giroux also finds conditions under which a convex surface has a tight $I-$ invariant contact neighborhood.

Theorem 2.3 (Giroux) If $\Sigma \neq S^{2}$ is a convex surface in a contact manifold $(M, \xi)$, then $\Sigma$ has a tight neighborhood if and only if no component of $\Gamma_{\Sigma}$ is null-homotopic in $\Sigma$. If $\Sigma=S^{2}, \Sigma$ has a tight neighborhood if and only if $\# \Gamma_{\Sigma}=1$.

We say that a contact structure on a manifold $M$ with boundary $\partial M$ is a contact structure with convex boundary if there is a contact vector field $v$ on $M$ transverse to $\partial M$. The following definition records the information about a contact structure near its convex boundary, but forgets the structure in the interior.

Definition 2.4 A convex structure is a quadruple $\left(M, \Gamma, R_{-}(\Gamma), R_{+}(\Gamma)\right)$ where $M$ is a compact oriented 3-manifold with nonempty boundary, $\Gamma$ is a disjoint union of simple closed curves contained in $\partial M$ nonempty on each component of $\partial M$, and $\partial M=R_{+}(\Gamma) \cup R_{-}(\Gamma), R_{+}(\Gamma) \cap R_{-}(\Gamma)=\Gamma$. Moreover $R_{+}(\Gamma), R_{-}(\Gamma)$ and $\Gamma$ are oriented so that the orientation of $R_{+}(\Gamma)$ agrees with the orientation induced on $\partial M$ by the orientation of $M$, and the orientation on $R_{-}(\Gamma)$ is the opposite one. $\Gamma$ is oriented in such a way that if $\alpha \subset \partial M$ is an oriented arc with $\partial \alpha \subset R_{+}(\Gamma) \cup R_{-}(\Gamma)$ that intersects $\Gamma$ transversely in one point and if $\Gamma \cdot \alpha=1$ then $\alpha$ must start in $R_{-}(\Gamma)$ and end in $R_{+}(\Gamma)$.

A contact structure on $M$ with convex boundary and a choice of a contact vector field $v$ such that $v$ is an oriented normal to $\partial M$ induces a convex structure on $M . \Gamma$ is defined to be the dividing set of $\partial M$ with respect to $v$, and $R_{+}(\Gamma)$ and $R_{-}(\Gamma)$ are the regions of $\partial M$ where the oriented normal vector $n_{\xi}$ to the contact planes and $v$ satisfy $n_{\xi} \cdot v>0$ and $n_{\xi} \cdot v<0$ respectively.

Definition 2.5 A convex structure $\left(M, \Gamma, R_{-}(\Gamma), R_{+}(\Gamma)\right)$ carries a tight contact structure if there is a tight contact structure on $M$, and a contact vector field $v$ such that $v$ is an oriented normal for $\partial M$ and both $\Gamma, R_{-}(\Gamma)$ and $R_{+}(\Gamma)$ are defined by $v$ as above. 
Note that if we change the orientation of the contact plane field $\xi, R_{-}(\Gamma)$ and $R_{+}(\Gamma)$ will switch.

\section{Legendrian curves on convex surfaces}

A Legendrian curve $C$ and the oriented normal to $\xi$ determine a framing along $C$. If $F r$ is another framing we define the twisting number $t(C, F r)$ as the relative framing between the one determined by the oriented normal to $\xi$ and $F r$. If $C$ lies on a surface $\Sigma, t(C, \Sigma)$ is defined to be the twisting number with respect to the framing defined on $C$ by $\Sigma$. Observe that if if $C$ is a Legendrian curve on a convex surface $\Sigma$, then its twisting number $t(C, \Sigma)$ is equal $\frac{1}{2} \#\left(C \cap \Gamma_{\Sigma}\right)$, where $\#\left(C \cap \Gamma_{\Sigma}\right)$ denotes the geometric intersection number. In fact it is easy to show the following.

Proposition 3.1 Let $C$ be a Legendrian curve on a convex surface $\Sigma$ with $t(C, \Sigma)=-n$. Then, after a small perturbation of $\Sigma$, there are local coordinates $(x, y, z)$ so that a neighborhood of $C$ in $M$ is isomorphic to the neighborhood $N=\left\{(x, y, z) \mid x^{2}+y^{2} \leq \varepsilon\right\}$ in $\mathbb{R}^{2} \times(\mathbb{R} / \mathbb{Z})$, where the set $x=0$ corresponds to $\Sigma, C$ is given by $x=y=0$, and the contact structure is determined by the 1-form $\alpha=\sin (2 \pi n z) d x+\cos (2 \pi n z) d y$. If the contact vector field determining the dividing set $\Gamma_{\Sigma}$ is $v=\frac{\partial}{\partial x}$, the dividing set is $\Gamma_{\Sigma}=\left\{\left(0, y, \frac{k}{2 n}\right) \mid 0 \leq k \leq 2 n\right\}$.

It is a standard fact that any curve in a contact manifold has in its isotopy class a nearby Legendrian curve. However, even more is true: this can be achieved even when we require the curve to lie on a convex surface isotopic to a fixed one and with the same dividing set. Let us call a union of closed curves $C$ on a convex surface $\Sigma$ nonisolating if (1) $C$ is transverse to $\Gamma_{\Sigma}$, and (2) every component of $\Sigma \backslash\left(\Gamma_{\Sigma} \cup C\right)$ has a boundary component which intersects $\Gamma_{\Sigma}$. Clearly this will be satisfied if every component of $C$ intersects $\Gamma_{\Sigma}$.

Theorem 3.2 (Legendrian Realization Principle [17]) Let $C$ be a nonisolating collection of closed curves on a convex surface $\Sigma$. Then there exists an admissible isotopy $\phi_{t}, t \in[0,1]$, so that

(1) $\phi_{0}=i d$,

(2) $\phi_{t}(\Sigma)$ are all convex,

(3) $\phi_{1}\left(\Gamma_{\Sigma}\right)=\Gamma_{\phi_{1}(\Sigma)}$,

(4) $\phi_{1}(C)$ is Legendrian. 
It follows that a nonisolating collection $C$ can be realized by a Legendrian collection $C^{\prime}$ with the same number of geometric intersections with $\Gamma_{\Sigma}$. A special case of this theorem, observed by Kanda, is the following:

Corollary 3.3 (Kanda) If $C$ is a closed curve in $\Sigma$ such that $C \pitchfork \Gamma_{\Sigma}$ and $C \cap \Gamma_{\Sigma} \neq \emptyset$, then $C$ can be realized as a Legendrian curve (in the sense of Theorem 3.2).

Giroux [12] proved that a closed oriented embedded surface can be deformed through a $C^{\infty}$-small isotopy to a convex surface. The following relative version is proven in Honda [17].

Theorem 3.4 (Existence of Convex Surfaces) Let $T \subset M$ be a compact, oriented, properly embedded surface with Legendrian boundary such that $t(C, T)$ $\leq 0$ for all components $C$ of $\partial T$. There exists a $C^{0}$-small isotopy of $T$, which is the identity on $\partial T$, that takes $T$ to a convex surface. The isotopy may be chosen to be $C^{\infty}$ outside of a small neighborhood of $\partial T$.

\section{Convex decompositions}

A 3-manifold $M$ is irreducible if every embedded 2-sphere $S^{2}$ bounds a 3-ball $B^{3}$. A properly embedded surface $\Sigma \subset M$ is incompressible if it contains no compressing disk, ie, an embedded disk $D \subset M$ with $D \cap \Sigma=\partial D$ which is homotopically nontrivial in $\Sigma$. A Haken decomposition of a $3-$ manifold $M$ is a sequence

$$
M=M_{0} \stackrel{S_{1}}{\rightsquigarrow} M_{1} \stackrel{S_{2}}{\rightsquigarrow} \ldots \stackrel{S_{n}}{\rightsquigarrow} M_{n},
$$

where $S_{i+1}$ is an incompressible surface in $M_{i}, M_{i+1}=M_{i} \backslash S_{i+1}$, and $M_{n}$ is a disjoint union of balls. Haken manifolds are 3-manifolds which admit Haken decompositions. Therefore, inductive arguments can often be applied to Haken manifolds. An irreducible manifold with non-empty boundary always has a Haken decomposition [20]. The idea we are pursuing in this paper is that when $M$ has a contact structure, and we choose the splitting surfaces to be convex, the information about the contact structure on $M$ can be recovered from the contact structure on the cut-up manifold $M \backslash S$ and the information contained in the dividing set on the splitting surface $S$. In this section we will describe how to perform convex splittings in the contact category.

When $(M, \xi)$ is a contact structure with convex boundary, we can choose a Haken decomposition of $(M, \partial M)$ to be, at each step, performed along incompressible surfaces with boundary $(S, \partial S)$ properly embedded in $(M, \partial M)$. At 
each step of the decomposition, we will do the same three things: perturb the cutting surface $(S, \partial S)$ to a convex surface with Legendrian boundary, cut $(M, \xi)$ along $S$ to obtain a manifold with corners $M \backslash S$ which inherits the restriction $\left.\xi\right|_{M \backslash S}$ of $\xi$, and finally round corners to obtain a smooth manifold and a contact structure with convex boundary on it.

We first need to perturb $\partial S$. We isotop each component $C$ of $\partial S \subset \partial M$ so that the geometric intersection $\#\left(C \cap \Gamma_{\partial M}\right)$ is minimized, provided this number is $\geq 2$. If the minimum geometric intersection is 0 , we can choose $C$ so $\#\left(C \cap \Gamma_{\partial M}\right)=2$, since every component of $\partial M$ nontrivially intersects $\Gamma_{\partial M}$. We artificially force the extra intersections because cutting along Legendrian curves with twisting number 0 is not as easy to control. Now we can use the Legendrian Realization Principle (Theorem 3.2) to make $\partial S$ Legendrian. Once we have prepared $\partial S$ as above, we perturb the surface $S$ so that near the boundary it is convex and the local picture is as in Figure 1.

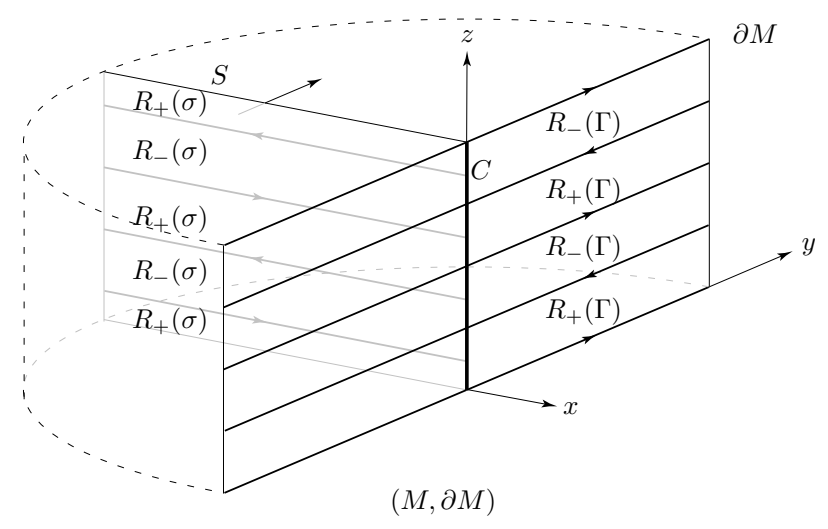

Figure 1

If $C$ intersects the dividing set $\Gamma_{\partial M}$ geometrically $2 n$ times, there is a neighborhood of $C$ in $M$ and local coordinates $(x, y, z)$ on it isomorphic to $N=$ $\left\{(x, y, z) \mid x^{2}+y^{2}<\varepsilon, x \leq 0\right\}$ in $\mathbb{R}^{2} \times(\mathbb{R} / \mathbb{Z})$ where the set $A=\{(x, y, z) \in$ $N \mid x=0\}$ corresponds to an annular neighborhood of $C$ in $\partial M$ and $B=$ $\{(x, y, z) \in N \mid y=0\}$ to an annular neighborhood of $C$ in $S$, and the 1form $\alpha=\sin (2 \pi n z) d x+\cos (2 \pi n z) d y$ determines the contact structure. If we choose the contact vector fields for $\partial M$ and $S$ in these coordinates to be respectively $v_{\partial M}=\frac{\partial}{\partial x}$ and $v_{S}=\frac{\partial}{\partial y}$ it is easy to calculate that the dividing sets are $\left.\Gamma_{\partial M}=\left\{0, y, \frac{k}{2 n}\right) \mid 0 \leq k<2 n\right\}$ and $\Gamma_{S}=\left\{(x, 0), \frac{1+2 k}{4 n} \mid 0 \leq k<2 n\right\}$.

If $\left(M, \Gamma, R_{+}, R_{-}\right)$is the convex structure associated to a contact structure $\xi$ with convex boundary, and if $S$ is a convex surface with Legendrian boundary 
properly embedded in $M$ and transverse to $\Gamma$, then the convex vector field $v_{S}$ given by $\frac{\partial}{\partial y}$ in the local coordinates discussed above can be extended to a convex vector field on $S$, which will determine a dividing set $\sigma$ on $S$ as well as subsets $R_{-}(\sigma)$ and $R_{+}(\sigma)$, defined as in the case of a closed surface.

The next definition abstracts the properties of a properly embedded convex surface with Legendrian boundary in a contact manifold with convex boundary.

Definition 4.1 A surface with divides $\left(S, \sigma, R_{+}(\sigma), R_{-}(\sigma)\right)$ is a compact oriented surface $S$, possibly with boundary, together with a disjoint collection of properly embedded arcs and simple closed curves $\sigma$ and a decomposition into two subsurfaces $S=R_{+}(\sigma) \cup R_{-}(\sigma), R_{+}(\sigma) \cap R_{-}(\sigma)=\sigma$. The orientation on $R_{+}(\sigma)$ is the orientation induced from $S$ while $R_{-}(\sigma)$ has the opposite orientation. The components of $\sigma$ are oriented so that if $\alpha \subset S$ is an oriented arc which intersects $\sigma$ transversely in one point and $\sigma \cdot \alpha=1$ then $\alpha$ starts in $R_{-}(\sigma)$ and ends in $R_{+}(\sigma)$.

Dividing curves on convex surfaces in tight contact manifolds satisfy special properties, as we saw in Theorem 2.3. For a convex surface with Legendrian boundary we have the following generalization:

Proposition 4.2 Let $(M, \xi)$ be a tight contact manifold with convex boundary, and let $\sigma$ be the dividing set of a convex surface $S$ with Legendrian boundary $\partial S$ transverse to the dividing set $\Gamma_{\partial M}$, such that every component of $\partial S$ intersects $\Gamma_{\partial M}$. Then $\sigma$ satisfies the following:

(1) On each component of $\partial S$ the points of $\sigma \cap \partial S$ alternate with the points of $\Gamma \cap \partial S$.

(2) The orientation on each arc of $\sigma$ is from $R_{-}(\Gamma)$ to $R_{+}(\Gamma)$.

(3) No closed curve in $\sigma$ bounds a disk in $S$.

Proof Parts 1 and 2 follow from the local coordinates picture discussed above and part 3 from Theorem 2.3.

When we split $(M, \partial M)$ along $(S, \partial S)$ we obtain a manifold with corners $M \backslash S$. To smooth the corners we use the following "corner-rounding" procedure. Each of the halves of $N$,

$$
N_{-}=\{(x, y, z) \in N \mid y \leq 0\}
$$

and

$$
N_{+}=\{(x, y, z) \in N \mid y \geq 0\}
$$


is replaced by the corresponding

$$
N_{-}^{r}=\left\{(x, y, z) \in N_{-} \mid x \leq \frac{-\varepsilon}{2} \text { or } \mathrm{y} \leq-\frac{\varepsilon}{2}+\sqrt{\frac{\varepsilon^{2}}{4}-\left(\mathrm{x}+\frac{\varepsilon}{2}\right)^{2}}\right\}
$$

and

$$
N_{+}^{r}=\left\{(x, y, z) \in N_{+} \mid x \leq \frac{-\varepsilon}{2} \text { or } \quad \mathrm{y} \leq+\frac{\varepsilon}{2}+\sqrt{\frac{\varepsilon^{2}}{4}-\left(\mathrm{x}+\frac{\varepsilon}{2}\right)^{2}}\right\} .
$$

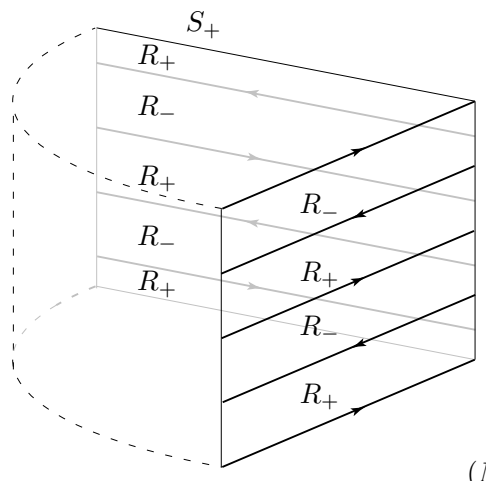

$(M \backslash S, \partial M \backslash \partial S)$

Figure 2

A quick look at the form $\alpha=\sin (2 \pi n z) d x+\cos (2 \sin z) d y$ determining $\xi$ and the normal vectors of the boundaries show, even without calculation, that the dividing set on the rounded boundary will be as in Figure 3. Clearly, ker $\alpha=$ $\operatorname{span}\left\{\frac{\partial}{\partial z}, \cos (2 \pi n z) \frac{\partial}{\partial x}-\sin (2 \pi n z) \frac{\partial}{\partial y}\right\}$, and the contact vector fields all lie in the $(x, y)$-plane. It is an easy calculation to see that when the contact vector rotates counterclockwise in the $(x, y)$-plane, the $z$-coordinate of the dividing set decreases.

We introduce the notion of a convex splitting to formalize the proces of obtaining the convex structure on the manifold with boundary $(M \backslash S, \partial M \backslash \partial S)$ by cutting $(M, \partial M)$ along the properly embedded convex surface with Legendrian boundary $S$, rounding the corners and looking at the new dividing set.

Definition 4.3 Let $(S, \sigma)$ be a surface with divides that is properly embedded in a convex structure $(M, \Gamma)$ so that $S$ and $\sigma$ are both transverse to $\Gamma$, and so that they satisfy properties $1-3$ listed above. We say that $(S, \sigma)$ defines a convex splitting $(M, \Gamma) \stackrel{(S, \sigma)}{\sim}\left(M^{\prime}, \Gamma^{\prime}\right) . M^{\prime}$ is $M$ split along $S$ and is denoted $M^{\prime}=M \backslash S . \partial M^{\prime}$ contains two disjoint copies of $S$ which are denoted $S_{+}$ and $S_{-} . S_{+}$are the components such that the outward orientation it inherits 

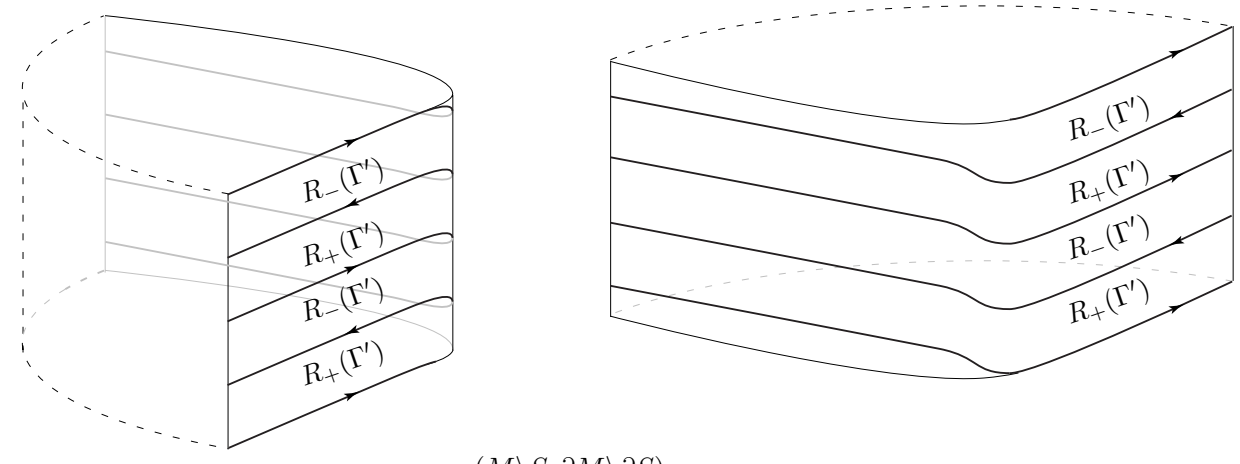

$(M \backslash S, \partial M \backslash \partial S)$

Figure 3

from $M^{\prime}$ agrees with the original orientation on $S$. Given a subset $X \subset S$ denote by $X_{+}$the corresponding subset of $S_{+}$, and similarly for $X_{-}$. Thus $\sigma_{+},\left(R_{+}(\sigma)\right)_{+},\left(R_{-}(\sigma)\right)_{+}$are all subsets of $S_{+}$. Define

$$
\begin{aligned}
R_{+}\left(\Gamma^{\prime}\right) & =\left(R_{+}(\Gamma) \backslash \partial S\right) \cup\left(R_{+}(\sigma)\right)_{+} \cup\left(R_{-}(\sigma)\right)_{-} \\
R_{-}\left(\Gamma^{\prime}\right) & =\left(R_{-}(\Gamma) \backslash \partial S\right) \cup\left(R_{-}(\sigma)\right)_{+} \cup\left(R_{+}(\sigma)\right)_{-} \\
\Gamma^{\prime} & =R_{+}\left(\Gamma^{\prime}\right) \cap R_{-}\left(\Gamma^{\prime}\right) .
\end{aligned}
$$

Finally, smooth all corners so that $\partial M^{\prime}$ is a smooth subset of $M^{\prime}$ and $\Gamma^{\prime}$ is a smooth subset of $\partial M^{\prime}$.

If we perform a Haken decomposition of a tight contact manifold with convex boundary along embedded convex surfaces with Legendrian boundary, rounding corners at each step along the way, we obtain in the end a disjoint union of spheres with tight contact structures on them. The following facts now come into play:

Proposition 4.4 Let $\xi$ be a tight contact structure on $B^{3}$ with convex boundary. Then $\# \Gamma_{\partial B^{3}}=1$.

This is just Theorem 2.3 restated.

Theorem 4.5 (Eliashberg [7]) Let $\xi$ be a contact structure on a neighborhood of $\partial B^{3}$ for which $\partial B^{3}$ is convex and $\# \Gamma_{\partial B^{3}}=1$. Then there exists a unique extension of $\xi$ to a tight contact structure on $B^{3}$, up to an isotopy which fixes the boundary. 


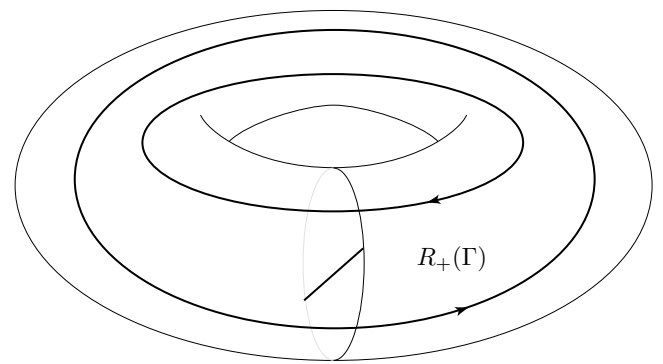

$(M, \Gamma)$

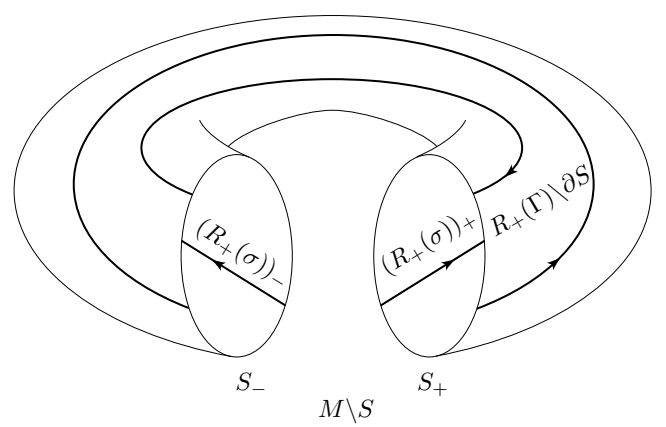

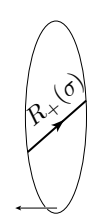

$(S, \sigma)$

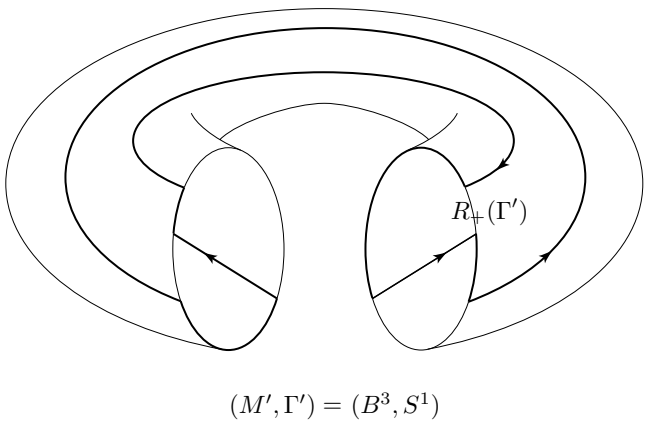

Figure 4

The decomposition of tight contact manifolds motivates the following definition of decomposability of convex structures.

Definition 4.6 A convex structure $(M, \Gamma)$ is decomposable if there exists a sequence of convex splittings

$$
(M, \Gamma) \stackrel{\left(S_{1}, \sigma_{1}\right)}{\rightsquigarrow}\left(M_{1}, \Gamma_{1}\right) \rightsquigarrow \cdots \stackrel{\left(S_{n}, \sigma_{n}\right)}{\rightsquigarrow}\left(M_{n}, \Gamma_{n}\right)
$$

such that $\left(M_{n}, \Gamma_{n}\right)$ is a disjoint union of $\left(B^{3}, S^{1}\right)$ 's.

We then have the following:

Theorem 4.7 If $(M, \Gamma)$ carries a tight contact structure, then it is decomposable.

Proof Let $\xi$ be a tight contact structure on $M$ which is adapted to $\Gamma$. Consider the Haken decomposition

$$
M=M_{0} \stackrel{S_{1}}{\rightsquigarrow} M_{1} \stackrel{S_{2}}{\rightsquigarrow} \ldots \stackrel{S_{n}}{\rightsquigarrow} M_{n} .
$$


Let $\Gamma_{0}=\Gamma$. Assume we have already performed convex splittings along convex surfaces with Legendrian boundary, so that we have $\left(M_{i}, \Gamma_{i}\right)$. In order to split along $S_{i+1}$ in a convex manner, make $\partial S_{i+1}$ Legendrian using the Legendrian Realization Principle, perturb $S_{i+1}$ so it is convex with Legendrian boundary, form $M_{i} \backslash S_{i+1}$, and round the corners. This yields $\left(M_{i+1}, \Gamma_{i+1}\right)$. Since $M$ is Haken, we eventually find that $M_{n}=\cup B^{3}$. Proposition 4.4 implies that for each $B^{3}$ we have $\# \Gamma_{\partial B^{3}}=1$.

Corollary 4.8 If $(M, \Gamma)$ carries a tight contact structure, then $\chi\left(R_{+}(\Gamma)\right)=$ $\chi\left(R_{-}(\Gamma)\right)$.

Proof If $(M, \Gamma) \stackrel{(S, \sigma)}{\sim}\left(M^{\prime}, \Gamma^{\prime}\right)$, then a computation shows that $\chi\left(R_{ \pm}\left(\Gamma^{\prime}\right)\right)=$ $\chi\left(R_{ \pm}(\Gamma)\right)+\chi(S)$. The result follows by induction on the length of the decomposition sequence for $(M, \Gamma)$.

\section{Sutured vs convex decompositions}

We now recall basic definitions from Gabai's theory of sutured manifolds [11]. It will be immediately obvious that they resemble the definitions just made. The point of this paper is to exploit the equivalence of basic notions in these theories.

Definition 5.1 A sutured manifold $(M, \gamma)$ is a compact oriented 3-manifold $M$ together with a set $\gamma \subset \partial M$ of pairwise disjoint annuli $A(\gamma)$ and tori $T(\gamma)$. $R(\gamma)$ denotes $\partial M \backslash \operatorname{int}(\gamma)$. Each component of $R(\gamma)$ is oriented. $R_{+}(\gamma)$ is defined to be those components of $R(\gamma)$ whose normal vectors point out of $M$ and $R_{-}(\gamma)$ is defined to be $R(\gamma) \backslash R_{+}(\gamma)$. Each component of $A(\gamma)$ contains a suture, ie, a homologically nontrivial oriented simple closed curve. The set of sutures is denoted $s(\gamma)$. The orientation on $R_{+}(\gamma), R_{-}(\gamma)$ and $s(\gamma)$ are related as follows. If $\alpha \subset \partial M$ is an oriented arc with $\partial \alpha \subset R(\gamma)$ that intersects $s(\gamma)$ transversely in one point and if $s(\gamma) \cdot \alpha=1$, then $\alpha$ must start in $R_{-}(\gamma)$ and end in $R_{+}(\gamma)$.

Definition 5.2 A sutured manifold with annular sutures is a sutured manifold $(M, \gamma)$ which satisfies the following:

(1) Every component of $M$ has nonempty boundary.

(2) Every component of $\partial M$ contains a suture. 
(3) Every component of $\gamma$ is an annulus.

Note that a sutured manifold $(M, \gamma)$ with annular sutures determines, and is determined by, the associated convex structure $(M, \Gamma)$ where $\Gamma=s(\gamma)$.

The definition of a sutured manifold splitting $(M, \gamma) \stackrel{S}{\rightsquigarrow}\left(M^{\prime}, \gamma^{\prime}\right)$ is quite similar to the definition of a convex splitting. However, unlike convex splittings, we do not have dividing curves to prescribe on the splitting surface $S$.

Assume $S$ is a properly embedded, oriented surface in $M$ such that:

(1) $\partial S \pitchfork \gamma$.

(2) If $S$ intersects an annular suture $A$ in arcs, then no such arc separates $A$.

(3) If $S$ intersects an annular suture $A$ in circles, then each such circle, with orientation induced from $S$, is homologous in $A$ to the oriented core $s(\gamma) \cap A$.

(4) If $S$ intersects a toroidal suture $T$ in circles, then no such circle is nullhomologous in $T$, and any two such circles, with orientations induced from $S$, are homologous in $T$.

(5) No component of $S$ is a disk $D$ with $\partial D \subset R(\gamma)$.

(6) No component of $\partial S$ bounds a disk in $R(\gamma)$.

Let $M^{\prime}=M \backslash S$ and let $S_{+}$and $S_{-}$be the copies of $S$ contained in $M^{\prime}$ where the orientation induced by $S$ points, respectively, out of and into $M^{\prime}$. As a first approximation, let $R_{ \pm}^{\prime}\left(\gamma^{\prime}\right)$ be $\left(R_{ \pm}(\gamma) \backslash S\right) \cup S_{ \pm} \cdot \gamma^{\prime}$ is supposed to separate $R_{+}^{\prime}\left(\gamma^{\prime}\right)$ and $R_{-}^{\prime}\left(\gamma^{\prime}\right)$ so define it to be the union of $\gamma \backslash S$ and $R_{+}^{\prime}\left(\gamma^{\prime}\right) \cap R_{-}^{\prime}\left(\gamma^{\prime}\right)$. Since $\gamma^{\prime}$ is supposed to be a union of annuli and tori, the actual definition of $\gamma^{\prime}$ is a union of $\gamma \backslash S$ and a regular neighborhood of $R_{+}^{\prime}\left(\gamma^{\prime}\right) \cap R_{-}^{\prime}\left(\gamma^{\prime}\right)$ and then $R_{ \pm}^{\prime}\left(\gamma^{\prime}\right)$ are shrunk by a corresponding amount.

Definition 5.3 A transversely oriented codimension- 1 foliation $\mathcal{F}$ is carried by $(M, \gamma)$ if $\mathcal{F}$ is transverse to $\gamma$ and tangent to $R(\gamma)$ with the normal direction pointing outward along $R_{+}(\gamma)$ and inward along $R_{-}(\gamma)$, and $\mathcal{F} \mid \gamma$ has no Reeb components. $\mathcal{F}$ is taut if each leaf of $\mathcal{F}$ intersects some closed curve or properly embedded arc connecting from $R_{-}(\gamma)$ to $R_{+}(\gamma)$ that is transverse to $\mathcal{F}$.

Let $S$ be a compact oriented surface with components $S_{1}, \ldots, S_{n}$. The Thurston norm of $S$ is defined to be

$$
x(S)=\sum_{i \text { such that } \chi\left(S_{\mathrm{i}}\right)<0}\left|\chi\left(S_{i}\right)\right| .
$$


Thus components with positive Euler characteristic, namely disks and spheres, do not contribute to the Thurston norm.

Definition 5.4 A sutured manifold $(M, \gamma)$ is taut if

(1) $M$ is irreducible.

(2) $R(\gamma)$ is norm-minimizing in $H_{2}(M, \gamma)$, that is if $S$ is an embedded surface in $M$ with $[S]=[R(\gamma)] \in H_{2}(M, \gamma)$ then $x(R(\gamma)) \leq x(S)$.

(3) $R(\gamma)$ is incompressible in $M$.

Except in a few cases, 2 implies 3. The reason is that compressions are normdecreasing unless the surface being compressed is an annulus. Thus 3 is meant to exclude the case that $M=B^{3}$ and $s(\gamma)$ consists of more than one component or that $M=D^{2} \times S^{1}$ and $s(\gamma)$ is compressible.

This definition of tautness of the sutured manifold is made because of the following theorem which is due to Gabai [11] and Thurston [28].

Theorem 5.5 A sutured manifold $(M, \gamma)$ is taut if and only if it carries a transversely oriented, taut, codimension-1 foliation $\mathcal{F}$.

The following correspondence shows that a sutured manifold splitting is a special case of the convex splitting:

(1) The cores of annular components of $\gamma$ can be viewed as dividing curves. If $\mathrm{T}$ is a toroidal component of $\gamma$ then just before cutting along a surface $S$ which intersects $T$ we substitute $T$ by $T$ with a pair of parallel homotopically nontrivial dividing curves, each of which has algebraic intersection 1 with each component of $S \cap T$.

(2) A component $\Sigma \subset \partial M$ may not have a suture at all, whereas a dividing set must not be empty. We remedy this by placing a pair of parallel homotopically nontrivial dividing curves on $\Sigma$ before cutting.

(3) Let $S$ be a cutting surface - realize the boundary as a Legendrian curve with twisting number $\leq-2$ - and choose $\Gamma_{S}$ so that every dividing curve is an arc which is $\partial$-compressible.

(4) When $M$ is cut along $S$ and rounded, all the dividing curves, except perhaps for the $T^{2}$ components and components $\Sigma \subset M$ without sutures, correspond to sutures. 


\section{Main Theorem}

Theorem 6.1 Let $(M, \gamma)$ be an irreducible sutured manifold with annular sutures, and let $(M, \Gamma)$ be the associated convex structure. The following are equivalent.

(1) $(M, \gamma)$ is taut.

(2) $(M, \gamma)$ carries a taut foliation.

(3) $(M, \Gamma)$ carries a universally tight contact structure.

(4) $(M, \Gamma)$ carries a tight contact structure.

Proof Without loss of generality we assume $M$ is connected.

$(1) \Rightarrow(2)$ is Gabai's theorem [11]. Gabai's theorem does not require the assumption that $(M, \gamma)$ have annular sutures.

$(2) \Rightarrow(1)$ by Thurston [28] does not require this assumption either.

$(2) \Rightarrow(3)$ is due to Eliashberg and Thurston [10] in the closed case. That their work can be applied in this context is the content of Theorem 6.2.

$(3) \Rightarrow(4)$ is immediate.

(4) $\Rightarrow(1)$ follows from Theorem 6.7. The assumption that $\partial M \neq \emptyset$ is crucial here. For by Bennequin [1] $S^{3}$ has a tight contact structure, but by Novikov [27] it has no taut foliation. Also the irreducibility of $M$ is necessary, since connect summing preserve tightness $([25],[2])$, whereas the universal cover of a taut foliation is $\mathbb{R}^{3}$.

\subsection{Confoliations}

In this section we will prove the following theorem:

Theorem 6.2 Let $\xi$ be a (finite depth) taut foliation which is carried by a sutured manifold $(M, \gamma)$ with annular sutures. Then there exists a modification of $\xi$ into a positive tight contact structure $\xi_{+}$such that $\partial M$ is convex and $\Gamma_{\partial M}=s(\gamma)$.

Before we begin the proof, we recall several notions from the theory of confoliations [10]. A positive confoliation $\xi$ is an oriented 2-plane field distribution on $M$ given by a 1 -form $\alpha$ which satisfies $\alpha \wedge d \alpha \geq 0$. The contact part of $\xi$ 
is $H(\xi)=\{x \in M \mid \alpha \wedge d \alpha>0\}$. For a subset $A \subset M$, the saturation $\hat{A}$ of $A$ is the subset of $M$ which consists of points which can be connected to a point in $A$ via a path which is everywhere tangent to $\xi$. $\xi$ is said to be transitive if $\widehat{H(\xi)}=M$.

Proof The proof is almost identical to the perturbation result for closed manifolds due to Eliashberg and Thurston [10]. The difference is that we need to modify the boundary carefully, and the modification $\xi_{+}$is usually not a perturbation of $\xi$. Since $\xi$ is carried by $(M, \gamma), \partial M$ is best thought of as a manifold with corners, where $R_{ \pm}=R_{ \pm}(\gamma)$ are leaves of $\xi$ and the leaves of $\xi$ (and hence $\left.R_{ \pm}(\gamma)\right)$ are transverse to $\gamma$. In order to use symplectic filling techniques, we need to exercise a little care, and extend $M$ and $\xi$ to an open manifold with finite geometry at infinity.

Step 1 We first extend $\xi$ in two ways to $M_{1}=M \cup\left(R_{+} \times[0, \infty)\right) \cup\left(R_{-} \times\right.$ $[0, \infty))$, where $R_{+} \times\{0\}=R_{+}, R_{-} \times\{0\}=R_{-}, \partial M_{1}=\gamma^{\prime}$, and $\gamma^{\prime}=\gamma \cup$ $\left(\partial R_{+} \times[0, \infty)\right) \cup\left(\partial R_{-} \times[0, \infty)\right)$ is smooth. The first extension is to a foliation (still called $\xi$ ) and the second is to a positive confoliation $\xi^{\prime}$ which is contact on $R_{ \pm} \times(0, \infty)$. Let $t$ be the coordinate in the $[0, \infty)$-direction for $R_{+} \times[0, \infty)$. The extension to a foliation $\xi$ on $M_{1}$ is easy - on $R_{ \pm} \times(0, \infty)$, simply take ker $d t$. We now construct $\xi^{\prime}$.

Lemma 6.3 If $R_{+}$has nonempty boundary, then there exists a 1-form $\beta$ on $R_{+}$with $d \beta>0$, whose singular foliation given by $\operatorname{ker} \beta$ has isolated singularities and no closed orbits, and whose flow is transverse to $\partial R_{+}$.

Proof Start with a singular foliation $\mathcal{F}$ on $R_{+}$which satisfies the following:

(1) $\mathcal{F}$ is Morse-Smale and has no closed orbits,

(2) The singular set consist of elliptic points (sources) and hyperbolic points.

(3) $\mathcal{F}$ is oriented, and for one choice of orientation the flow is transverse to and exits from $\partial R_{+}$.

For example, a gradient-like vector field would do. Next, modify $\mathcal{F}$ near each of the singular points so that $\mathcal{F}$ is given by $\beta_{0}=y d x-x d y$ near an elliptic point and $\beta_{0}=y d x+2 x d y$ near a hyperbolic point. Therefore, we have $\mathcal{F}$ given by $\beta_{0}$ which satisfies $d \beta_{0}>0$ near the singular points. Now, let $\beta=f \beta_{0}$, where $f$ is a positive function with $d f(X)>>0$, and $X$ is an oriented vector field for $\mathcal{F}$ (nonzero away from the singular points). Since $d \beta=d f \wedge \beta_{0}+f d \beta_{0}$, $d f(X)>>0$ guarantees that $d \beta>0$. 
Choose a 1 -form $\beta$ on $R_{+}$as in the lemma. Consider the 1 -form $\alpha^{\prime}=d t+f(t) \beta$ on $R_{+} \times[0, \infty)$, where $f(0)=0, f(t)=1$ for $t \geq 1$, and $f(t)>0$ for $t>0$. $\alpha^{\prime} \wedge d \alpha^{\prime}=f(t) d t \wedge d \beta>0$ on $R_{+} \times(0, \infty)$, since $d \beta>0$. Therefore, $\alpha^{\prime}$ gives rise to an extension of $\xi^{\prime}$ to a positive confoliation on $M_{1}$. The construction is similar on $R_{-} \times[0, \infty) . \xi^{\prime}$ is foliated on $M$ and contact on $M_{1} \backslash M$.

Step 2 Next extend $\xi$ to a foliation and $\xi^{\prime}$ to a positive confoliation on $M_{2}=M_{1} \cup\left(\gamma^{\prime} \times[0, \infty)\right)$. Denote $\gamma^{\prime} \times\{0\}=\gamma^{\prime}$ and assign coordinates $(\theta, y, z)$ to $\gamma^{\prime} \times[0, \infty)=S^{1} \times \mathbb{R} \times[0, \infty)$ by setting $y= \pm(t+1)$ on $\mathbb{R}_{ \pm} \times[0, \infty)$ and $\gamma=S^{1} \times[-1,1] \subset \gamma^{\prime}$. Since $\left.\xi\right|_{\gamma}=\left.\xi^{\prime}\right|_{\gamma}$ has no Reeb components, we may assume that $\left.\frac{\partial}{\partial y} \pitchfork \xi\right|_{\gamma}$. This means that, on $\gamma^{\prime} \times\{0\}$, we can take the characteristic foliation for $\xi$ to be given by a 1-form $\alpha=d y-g(\theta, y, 0) d \theta$, where $g=0$ if $y \geq 1$ or $y \leq-1$. We extend $\alpha$ to a foliated 1 -form on $\gamma^{\prime} \times I$ by taking $\alpha=d y-g(\theta, y, 0) d \theta$. Next, on $\gamma^{\prime} \times\{0\}$, the characteristic foliation of $\xi^{\prime}$ is given by the 1 -form $\alpha^{\prime}=d y-h(\theta, y, 0) d \theta$, where $h<0$ for $y>1$ or $y<-1$, and $h$ is independent of $y$ for large positive or large negative $y$. Extend $\alpha^{\prime}$ to a positive confoliated 1 -form on $\gamma^{\prime} \times[0, \infty)$ by taking $h$ with $\frac{\partial h}{\partial z}<0$ and $\lim _{z \rightarrow \infty} h(\theta, y, z)=C$, where $C$ is a fixed large negative number. Therefore, we have a confoliation $\xi^{\prime}$ on $M_{2}$ whose contact part is $M_{2} \backslash M$.

Notice that if we took $M \cup\left(R_{ \pm} \times[0,1]\right) \cup\left(\gamma^{\prime \prime} \times[0, n]\right), n$ large, where $\gamma^{\prime \prime}=\left(\partial R_{+} \times\right.$ $[0,1]) \cup\left(\partial R_{-} \times[0,1]\right) \cup \gamma$, then we can round the corners to obtain a manifold with boundary $M_{3}$ (isotopic to $M$ if we ignore corners). The characteristic foliation on $\partial M_{3}$ is Morse-Smale, and $\Gamma_{\partial M_{3}}$ is isotopic to $s(\gamma)$.

Step 3 In this step we modify $\xi^{\prime}$ on $M_{2}$ (fixing $\xi^{\prime}$ on $M_{2} \backslash N(M)$, where $N(M)$ is a small neighborhood of $M)$ to obtain $\xi_{+}$which is contact on all of $M_{2}$. This step follows directly from Eliashberg and Thurston's argument [10]. We list the relevant results:

Proposition 6.4 Any $C^{2}$-confoliation can be $C^{0}$-approximated by a $C^{1}-$ smooth transitive confoliation.

Proposition 6.5 Any $C^{k}$-smooth transitive positive confoliation, $k \geq 1$, admits a $C^{k}$-close approximation by a positive contact structure.

It is easy to see that the propositions hold while fixing $\xi^{\prime}$ on $M_{2} \backslash N(M)$. Therefore, we obtain $\xi_{+}$which is a positive contact structure and agrees with $\xi^{\prime}$ 'at infinity'. 
Step 4 We prove that $\left(M_{2}, \xi_{+}\right)$is symplectically semi-fillable. We will construct a dominating 2-form $\omega$ for $\xi_{+}$(ie, a closed 2-form for which $\left.\omega\right|_{\xi_{+}}>0$ everywhere).

First recall the construction of a dominating 2-form $\omega$ on $M$ for the foliation $\xi$. Since the foliation $\xi$ is taut, through each point there exists a closed transversal or a transversal arc with endpoints on $R_{+}$and $R_{-}$. Let $\delta_{p}$ be a transversal through the point $p$ and $N_{p}$ be a tubular neighborhood of $\delta_{p}$. Then $N_{p}$ is foliated by an interval's worth or $S^{1}$ 's worth of disks, and we have a projection $\pi_{p}: N_{p} \rightarrow D_{p}$, where $D_{p}$ is a disk. Let $\omega_{p}$ be the closed 2-form $\pi_{p}^{*}\left(f_{p} A_{p}\right)$, where $A_{p}$ is an area form on $D_{p}$ and $f_{p}$ is a nonnegative function on $D_{p}$ with support inside $D_{p}$ and such that $f_{p}\left(\pi_{p}(p)\right)>0$. We may cover $M$ by $N_{p}$ so that $\bigcup_{p} \operatorname{supp}\left(\omega_{p}\right)=M$, and take a finite subcover. We would then take the dominating 2-form to be $\omega=\sum \omega_{p}$ (finite sum). Note that these $\omega_{p}$ are additive.

For our purposes, we need to control this construction more carefully. Let $M^{\prime}=M \cup\left(R_{+} \times[0, \varepsilon]\right) \cup\left(R_{-} \times[0, \varepsilon]\right)$. Extend the transversal arcs $\delta_{p}$ ending at $R_{ \pm}$on $M$ so that on $R_{ \pm} \times[0, \varepsilon]$ they restrict to $\{p t\} \times[0, \varepsilon]$, and choose $N_{p}$ so that $N_{p} \cap\left(R_{+} \times[0, \varepsilon]\right)=D_{p} \times[0, \varepsilon]$ (same for $R_{-}$). Therefore, on $R_{ \pm} \times[0, \varepsilon]$ we would have $\omega_{p}=\pi^{*}\left(g_{p} B_{p}\right)$, where $\pi: R_{ \pm} \times[0, \varepsilon] \rightarrow R_{ \pm}, B_{p}$ is some area form on $R_{ \pm}$and $g_{p}$ is a nonnegative function. $\omega$ would then have the property that $\omega=\pi^{*}(A)$, where $A$ is some area form for $R_{ \pm}$. Therefore we can extend $\omega$ to $M_{1}$ so that $\omega=\pi^{*}(A)$, where $\pi: R_{ \pm} \times[0, \infty) \rightarrow R_{ \pm}$is the first projection and $A$ is an area form for $R_{ \pm}$. We can further extend it to $M_{2}$ so that $\omega=d z d \theta$ on $\gamma^{\prime} \times[\varepsilon, \infty)$. Extending in the $M_{2}$-direction is easy if we took care to choose (1) $\delta_{p}$ to be $\operatorname{arcs}$ with $\theta=$ const. and $z=0$, if $p \subset \gamma^{\prime}$, and (2) $N_{p} \subset M^{\prime} \cup\left(\gamma^{\prime} \times[0, \varepsilon]\right)$. This means we can simply add the form $f(z) d z d \theta$, where $f(z)=1$ for $z \geq \varepsilon, f(0)=0$, and $f(z)>0$ for $z>0$. By our construction of $\xi_{+}$, the closed $2-$ form $\omega$ satisfies $\left.\omega\right|_{\xi_{+}}>0$ as well as $\left.\omega\right|_{\xi}>0$.

Define a closed 2-form $\widetilde{\omega}=\omega+d(s \alpha)$ on $M_{2} \times[-\varepsilon, \varepsilon]$, where $s$ is the variable for $[-\varepsilon, \varepsilon], \alpha$ is a nowhere zero 1 -form whose kernel is $\xi$, and $\varepsilon>0$ is small enough. Since we can obtain $\xi_{+}$positive and $\xi_{-}$negative (similarly), $\left(M_{2}, \xi_{+}\right)$ is symplectically semi-fillable and dominated by $\widetilde{\omega}$. We have the following symplectic semi-filling result:

Theorem 6.6 (Gromov-Eliashberg) Let $(X, \widetilde{\omega})$ be a (not necessarily compact) symplectic 4-manifold with contact boundary $(M, \xi)$ which satisfies $\left.\widetilde{\omega}\right|_{\xi}>$ 0 . Assume there exists a calibrated almost complex structure $J$ on $M$ which preserves $\xi$, and a corresponding Riemannian metric $g$ which has finite geometry at infinity, ie, 
(1) $g$ is complete,

(2) the sectional curvature of $g$ is bounded above, and

(3) the injectivity radius of $g$ is bounded below by some $\varepsilon>0$.

Then $(M, \xi)$ is a tight contact manifold.

By our construction, $M_{2} \times[-\varepsilon, \varepsilon]$ has finite geometry at infinity. Now pass to the universal cover of $M_{2} \times[-\varepsilon, \varepsilon]$, which also has finite geometry at infinity. Theorem 6.6 implies that $\xi_{+}$is universally tight. Hence so is $\xi_{+}$restricted to $M_{3}$.

Remark It is possible to prove that if $(M, \gamma)$ is taut, then $(M, \Gamma)$ carries a universally tight contact structure without resorting to symplectic filling. Instead we may use a convex decomposition which matches Gabai's sutured manifold decomposition, and prove a gluing theorem for tight contact structures. This will be carried out in [19], using ideas in [18].

\subsection{Proof of $(4) \Rightarrow(1)$}

Theorem 6.7 If $(M, \Gamma)$ carries a tight contact structure then $(M, \Gamma)$ is taut.

Proof Let us assume instead that there exists a surface $T \subset M$ such that

(1) $[T]=\left[R_{+}(\Gamma)\right]=\left[R_{-}(\Gamma)\right] \in H_{2}(M, \Gamma)$.

(2) $x(T)<x\left(R_{+}(\Gamma)\right)$.

The proof will follow from a sequence of lemmas and a calculation in the end.

Lemma 6.8 It is possible to modify $T$ so that $T$ satisfies (1), (2) as well as

(3) $\partial T=\Gamma$.

Proof Let $\Gamma_{0}$ be a connected component of $\Gamma$, and consider all the 'sheets' $T_{1}, \cdots, T_{m}$ of $T \cap N\left(\Gamma_{0}\right)$, where $N\left(\Gamma_{0}\right)$ is a small neighborhood of $\Gamma_{0}$. Since $\left[R_{+}(\Gamma)\right] \mapsto[\Gamma]$ under the boundary map $H_{2}(M, \Gamma) \stackrel{\partial}{\rightarrow} H_{1}(\Gamma)$, if $m>1$, then there must exist two consecutive sheets $T_{i}$ and $T_{i+1}$ which are oppositely oriented. In this case, we may surger $T$ by gluing $T_{i}$ and $T_{i+1}$ along $\Gamma_{0}$, rounding, and pushing the two sheets off of $\Gamma_{0}$. In this fashion we may reduce $m$ until it eventually becomes 1 . 
Lemma 6.9 In addition, we may take $T$ to satisfy

(4) $x(T)=-\chi(T)$.

Proof This is asking that $T$ have no disk or sphere components, which are the ones that contribute positively to the Euler characteristic but do not contribute to the Thurston norm. The irreducibility of $M$ assures us that every $S^{2}$ bounds a 3-ball, and can be removed from $T$ without affecting homology. We claim that there can be no disks $D$ with $\delta=\partial D$ which is a component of $\Gamma$, unless $(M, \Gamma)=\left(B^{3}, S^{1}\right)$. If there is such a disk $D$, then take a curve $\delta^{\prime} \subset \partial M$ parallel to $\delta$ which has no intersections with $\Gamma$. Use the Legendrian Realization Principle to realize $\delta^{\prime}$ as a Legendrian curve with $t\left(\delta^{\prime}, \partial M\right)=0 . \delta^{\prime}$ will then bound a disk $D^{\prime}$ with $t\left(\delta^{\prime}, D^{\prime}\right)=0$. This is an equivalent definition of the existence of an overtwisted disk. If $(M, \Gamma)=\left(B^{3}, S^{1}\right)$, Theorem 6.7 is immediate.

Lemma 6.10 In addition, $T$ may be modified so that

(5) $W$, the union of components $M \backslash T$ which intersect $R_{+}(\Gamma)$, satisfies $\partial W=$ $R_{+}(\Gamma) \cup T_{-}$.

Here, if $M^{\prime}=M \backslash T$, then we define $T_{+}, T_{-}$to be copies of $T$ contained in $M^{\prime}$, where the orientation induced by $T$ points out of and into $M^{\prime}$ (respectively).

Proof Define the function $\phi: M \backslash T \rightarrow \mathbb{Z}$ as follows. Assign $\phi\left(M_{0}\right)=0$, where $M_{0}$ is some connected component of $M \backslash T$ which borders $R_{-}(\Gamma)$. For another connected component $M_{i}$, take an arc $\alpha$ which starts in $M_{0}$ and ends in $M_{i}$, and define $\phi\left(M_{i}\right)=[\alpha \cap T]$. This number is independent of $\alpha$, for if $\alpha^{\prime}$ is another curve with the same endpoints, then $\left[\left(\alpha-\alpha^{\prime}\right) \cap T\right]=\left[\left(\alpha-\alpha^{\prime}\right) \cap R_{+}(\Gamma)\right]=0$. Note that all the components $M_{i}$ which border $\partial M$ have $\phi\left(M_{i}\right)$ equal to either 0 or 1 . If $\phi(M \backslash T) \neq\{0,1\}$, then choose $M_{i}$ with $\phi\left(M_{i}\right)$ extremal. $\partial M_{i}$ will not intersect $\partial M$ and will consist of components of $T$. Since these components bound $M_{i}$, we may throw them away without increasing $x(T)$. Thus we may assume $\phi(M \backslash T)=\{0,1\}$.

Let $M_{1}$ be a component of $M \backslash T$ which intersects $R_{+}(\Gamma)$ and let $\alpha$ be an arc which starts in $M_{0}$ and ends in $M_{1} \cap R_{+}(\Gamma)$. Then $\phi\left(M_{1}\right)=[\alpha \cap T]=$ $\left[\alpha \cap R_{+}(\Gamma)\right]=1 . M_{1} \cap R_{-}(\Gamma)=\emptyset$, for otherwise there exists an arc $\beta$ connecting points of $R_{+}(\Gamma)$ and $R_{-}(\Gamma)$ which doesn't intersect $T$. Also $M_{1} \cap T_{+}=\emptyset$, for crossing $T_{+}$increases $\phi$, and $\phi$ already takes its maximum value on $M_{1}$. It follows that $\partial W_{1} \subset R_{+}(\Gamma) \cup T_{-}$. 
Conversely, suppose that $M_{2}$ is a component of $M \backslash T$ which intersects $T_{-}$. Since crossing $T_{-}$decreases $\phi$, it follows that $\phi\left(M_{2}\right)=1 . M_{2} \cap R_{-}(\Gamma)=\emptyset$; otherwise following an arc from $R_{-}(\Gamma)$ to $R_{+}(\Gamma)$ would increase the value of $\phi$ by 1. Also $M_{2} \cap T_{+}=\emptyset$ since crossing $T_{+}$increases $\phi$. For $M_{2}$ to be included in $W$, we require that $M_{2}$ intersect $R_{+}(\Gamma)$. If this is not the case, then $\partial M_{2} \subset T_{-}$, and this component of $T$ can be eliminated from $T$.

Lemma 6.11 There exists an isotopy $\phi_{t}: T \rightarrow M, t \in[0,1]$, such that $\phi_{0}(T)=T, S \stackrel{\text { def }}{=} \phi_{1}(T)$ is a convex surface, and $\phi_{t}(\partial T), t \in[0,1]$, is contained in an annulus $N(\Gamma) \subset \partial M$ which contains $\Gamma$.

Proof By Lemma 6.8, $\partial T=\Gamma$. Perturb $T$ so that each component of $\partial T$ is transverse to and non-trivially intersects $\Gamma$. By the Legendrian Realization Principle we may assume $\partial T$ is a union of Legendrian curves. By Theorem 3.4, $T$ may be isotoped to a convex surface.

Completion of the proof of Theorem 6.7 Let $\left(M^{\prime}, \Gamma^{\prime}\right)$ denote $(M, \Gamma)$ split along $(S, \sigma)$, where $S$ is as in Lemma 6.11 and $\sigma$ is its dividing set. Also let $W$ be as in Lemma 6.10. Recall $\partial W=R_{+}(\Gamma) \cup T_{-}$. By our choice of $S$, $M \backslash S \cong M \backslash T$, and we denote the components of $M \backslash S$ which correspond to $W$ by $\bar{W}$. The convex structure on $\bar{W}$ is denoted $\left(\bar{W}, \bar{\Gamma}, R_{-}(\bar{\Gamma}), R_{+}(\bar{\Gamma})\right)$.

We must show how $R_{+}(\bar{\Gamma})$ is related to $R_{+}(\Gamma)$. Let $N(\Gamma)$ be a regular neighborhood of $\Gamma$ in $\partial M$ which contains the isotopy of $\partial T$ to $\partial S$. Let $R_{+}$be the closure of $R_{+}(\Gamma) \backslash N(\Gamma)$. It follows that $R_{+}$is contained in the interior of $R_{+}(\bar{\Gamma})$. It follows that there exist subsurfaces $A$ and $B$ of $\partial \bar{W}$ which intersect along circles such that

$$
\begin{gathered}
R_{+}(\bar{\Gamma})=R_{+} \cup A \\
R_{-}(\bar{\Gamma})=B \\
A \cup B \cong S_{-} \cong T_{-} .
\end{gathered}
$$

By Corollary 4.8, $\chi\left(R_{+}(\bar{\Gamma})\right)=\chi\left(R_{-}(\bar{\Gamma})\right)$. An argument similar to that of the proof of Lemma 6.9 gives $\chi(A) \leq 0$; thus it follows that

$$
\chi\left(R_{+}\right)=\chi(B)-\chi(A) \geq \chi(B)+\chi(A)=\chi\left(T_{-}\right) .
$$

Since $R_{+} \cong R_{+}(\Gamma)$ and $T_{-} \cong T$, it follows that $x\left(R_{+}(\Gamma)\right) \leq x(T)$.

Acknowledgements We thank the referee for helpful comments on improving the exposition. 


\section{References}

[1] D Bennequin, Entrelacements et équations de Pfaff, Astérisque, 107-108 (1983) 87-161

[2] V Colin, Chirurgies d'indice un et isotopies de sphères dans les variètès de contact tendues, C. R. Acad. Sci. Paris, Sr. I Math. 324 (1997) 659-663

[3] V Colin, Recollement de variétés de contact tendues, Bull. Soc. Math. France, 127 (1999) 43-69

[4] Y Eliashberg, Classification of overtwisted contact structures on 3-manifolds, Invent. Math. 98 (1989) 623-637

[5] Y Eliashberg, Topological characterization of Stein manifolds of dimension $>2$, Intern. Journal of Math. 1 (1990) 29-46

[6] Y Eliashberg, Filling by holomorphic discs and its applications, London Math. Soc. Lecture Note Series, 151 (1991) 45-67

[7] Y Eliashberg, Contact 3-manifolds twenty years since J Martinet's work, Ann. Inst. Fourier, 42 (1992) 165-192

[8] Y Eliashberg, Unique holomorphically fillable contact structure on the 3 -torus, Internat. Math. Res. Notices 2 (1996) 77-82

[9] J Etnyre, K Honda, On the non-existence of tight contact structures,

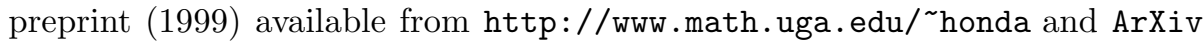
math.GT/9910115

[10] Y Eliashberg, W Thurston, Confoliations, University Lecture Series, 13, Amer. Math. Soc. Providence (1998)

[11] D Gabai, Foliations and the topology of 3-manifolds, J. Diff. Geom. 18 (1983) 445-503

[12] E Giroux, Convexité en topologie de contact, Comment. Math. Helvetici, 66 (1991) 637-677

[13] E Giroux, Une structure de contact, même tendue, est plus ou moins tordue, Ann. Scient. Ec. Norm. Sup. 27 (1994) 697-705

[14] E Giroux, Structures de contact en dimension trois et bifurcations des feuilletages de surfaces, preprint (1999)

[15] R Gompf, Handlebody construction of Stein surfaces, Annals of Math. 148 (1998) 619-693

[16] M Gromov, Pseudo-holomorphic curves in symplectic manifolds, Invent. Math. 82 (1985) 307-347

[17] K Honda, On the classification of tight contact structures I: lens spaces, solid tori, and $T^{2} \times I$, preprint (1999) revised version available from http: //www . math. uga.edu/ honda

[18] K Honda, Gluing tight contact structures, preprint (2000) available from http://www . math.uga.edu/ honda 
[19] K Honda, W H Kazez, G Matić, in preparation

[20] W Jaco, Lectures on three-manifold topology, CBMS Regional Conference Series in Mathematics, 43, American Mathematical Society, Providence, R.I. (1980)

[21] Y Kanda, The classification of tight contact structures on the 3-torus, Comm. in Anal. and Geom. 5 (1997) 413-438

[22] Y Kanda, On the Thurston-Bennequin invariant of Legendrian knots and non exactness of Bennequin's inequality, Invent. Math. 133 (1998) 227-242

[23] P Lisca, Symplectic fillings and positive scalar curvature, Geometry \& Topology, 2 (1998) 103-116

[24] P Lisca, G Matić, Stein 4-manifolds with boundary and contact structures, Top. and its App. 88 (1998) 55-66

[25] S Makar-Limanov, Morse surgeries of index 0 on tight manifolds, preprint (1997)

[26] J Martinet, Formes de contact sur les variétés de dimension 3, Springer Lecture Notes in Math. 209, 142-163

[27] S Novikov, Topology of foliations, Trans. Moscow Math. Soc. 14 (1963) 268305

[28] W Thurston, A norm for the homology of 3-manifolds, Mem. Amer. Math. Soc. 59 No. 339 (1986) 99-130

[29] I Torisu, Convex contact structures and fibered links in 3-manifolds, Internat. Math. Res. Notices (2000) No. 9, 441-454 